\title{
CONFÉRENCE DE CONSENSUS
}

\author{
NOMBRES ET OPÉRATIONS : \\ PREMIERS APPRENTISSAGES \\ À L'ÉCOLE PRIMAIRE
}

\section{RECOMMANDATIONS DU JURY}

26 NOVEMBRE 2015

Lycée Buffon (Paris XVè)

En partenariat avec : 
Les 12 et 13 novembre 2015 s'est tenue la deuxième conférence de consensus intitulée : "Nombres et opérations : premiers apprentissages à l'école primaire " organisée par le Cnesco et l'IFÉ/ENS de Lyon.

Une conférence de consensus, telle qu'elle est conçue par ses promoteurs (Cnesco et IFÉ/ENS de Lyon), vise à faire le lien entre, d'un côté, les préoccupations et les questions des praticiens et du grand public, et, de l'autre, les productions scientifiques.

\section{La conférence remplit deux fonctions majeures}

- Elle constitue une passerelle entre le monde de la recherche et les univers des praticiens et du grand public qui échangent autour des travaux de la recherche afin d'aboutir à des conclusions fondées scientifiquement. À l'issue de la conférence, le consensus se concrétise sous la forme de constats et de recommandations rédigés par un jury d'acteurs de terrain après l'audition d'experts.

- Elle agit comme un levier pour le changement dans le système éducatif français : ses résultats, largement diffusés dans la communauté éducative grâce à des partenariats multiples (Café Pédagogique, Canopé, ESENESR, Réseau des ÉSPÉ), permettent, à la fois, d'aider les parents dans leur rôle d'éducateur, et d'éclairer, dans leurs pratiques, les professionnels de l'Éducation.

\section{À quoi servent les recommandations du jury de la conférence ?}

\section{Comment sont rédigées les recommandations?}

Les dix-huit membres du jury de la conférence de consensus, après avoir pris connaissance de la recherche scientifique sur l'apprentissage de la numération et, lors des deux jours de séances publiques, écouté les experts ainsi que les praticiens, se sont réunis à huis clos pour aboutir, par consensus, à la rédaction de conclusions.

\section{Quelles sont les caractéristiques des recommandations?}

Les recommandations du jury présentent plusieurs caractéristiques. Elles visent à la fois une perspective temporelle longue en évoquant la modification des programmes et des manuels scolaires ou la formation des enseignants mais également de court terme en proposant de faire évoluer les pratiques des enseignants dans leur classe et en donnant des clés pour faciliter l'apprentissage des mathématiques aux parents d'élèves. 
Comment se poursuit le travail de recommandation de la conférence de consensus ?

Un dossier de ressources de la conférence est mis en ligne sur le site Internet du Cnesco: http://www.cnesco.fr/fr/conference-de-consensus-numeration/. Celui-ci permettra de diffuser aux professionnels de l'éducation un fond documentaire, les vidéos de présentation des experts ainsi que les recommandations du jury. Le Café Pédagogique sera un relais pour diffuser largement ces informations.

En partenariat avec l'ESENESR, Canopé et le Réseau des ESPÉ, la production de sessions d'information et de formations pour les professionnels de l'éducation sera étudiée.

Les recommandations pourront également être valorisées à travers par exemple la promotion d'une mallette pédagogique ainsi que lors d'événements en lien avec le sujet, comme "La semaine des Mathématiques ".

Enfin, le Cnesco et l'IFÉ/ENS de Lyon poursuivront leur travail en étudiant l'évolution des pratiques d'apprentissage des nombres et des opérations (étude qualitative) et suivront les enquêtes nationales et internationales sur les résultats des élèves. 
La France est réputée pour la qualité de sa recherche et de la formation de ses élites en mathématiques. Mais lorsque l'on s'intéresse aux compétences mathématiques de l'ensemble des élèves de l'école primaire, la situation est moins flatteuse et même, dans certains cas, préoccupante. Les évaluations des compétences mathématiques réalisées par l'Éducation nationale (Direction de l'évaluation, de la prospective et de la performance - DEPP -) dans différentes années scolaires mettent en évidence de sérieuses lacunes chez de nombreux élèves. Ainsi, seuls $58 \%$ des élèves maîtrisent les compétences attendues en fin de CM2. Plus de 40 \% des élèves n'ont pas acquis les connaissances de base relatives au calcul mental et aux opérations sur les grands nombres et les décimaux. Par exemple, seulement $27 \%$ des élèves de $\mathrm{CM} 2$ sont capables de déterminer le nombre à virgule correspondant à $1 / 4$; et à peine plus de la moitié de ces élèves $(51,6 \%)$ sont capable d'effectuer correctement la multiplication « 39 x 57 ». Ces observations, et d'autres encore, sont inquiétantes, car elles concernent des connaissances indispensables pour pouvoir aborder sans difficulté majeure les apprentissages mathématiques dans la suite du cursus scolaire. Ce sont aussi des connaissances fondamentales que doit posséder tout citoyen pour pouvoir faire face aux problèmes de la vie quotidienne. Ces lacunes ne se résorbent malheureusement pas dans la suite de la scolarité. L'enquête PISA (Programme international pour le suivi des acquis des élèves organisé par $I^{\prime} O C D E^{1}$ ) réalisée en 2012 sur les savoirs et les savoir-faire des élèves de 15 ans dans 65 pays montre qu'en mathématiques, la France se situe dans la moyenne des pays de I'OCDE $^{2}$. Derrière cette performance moyenne, on observe une grande disparité entre les élèves français. Si $12,9 \%$ parviennent à répondre aux questions les plus complexes (niveaux 5 et 6), 22,4 \% ne réussissent à traiter que les questions les plus simples (niveaux 1 et 2 ).

Les lacunes observées chez les élèves ont des origines multiples, souvent interdépendantes. Certaines sont liées aux conditions sociales et familiales des élèves. Ainsi, l'enquête PISA de 2012 montre qu'en France, 22,5\% des différences de performances entre élèves sont imputables au milieu socio-économique. De tous les pays de l'OCDE, la France est celui où les écarts de performances en fonction du milieu socio-économique sont les plus grands. D'autres facteurs à l'origine des lacunes d'apprentissage sont de nature pédagogique. Ces trente dernières années, les recommandations pédagogiques provenant de l'éducation nationale, des manuels scolaires ou encore des chercheurs ont régulièrement changé, laissant les enseignants sans direction claire face à leurs élèves. Il ne suffit pas de publier de nouveaux programmes pour résoudre ce type de problème. Les programmes doivent en effet être transposés dans les pratiques pédagogiques, ce qui est loin d'être évident. Les enseignants ont aujourd'hui besoin de conseils et de lignes directrices pour les aider à traduire les programmes en activités pédagogiques pertinentes, adaptées à un public scolaire diversifié. Le besoin de ces lignes directrices ne concerne pas seulement les enseignants, mais aussi les personnes susceptibles d'avoir une influence directe ou indirecte sur les pratiques pédagogiques (formateurs

${ }^{1}$ Organisation de coopération et de développement économiques.

${ }^{2}$ Notons que des pays comme la Suisse, les Pays-Bas, la Belgique ou le Canada, qui ne sont pas très éloignés de la France du point de vue socioéconomique et culturel, obtiennent des scores moyens en mathématiques supérieurs à la moyenne des pays de l’OCDE. 
d'enseignants, inspecteurs, auteurs de manuels et de didacticiels...). La cohérence entre ces différents acteurs est essentielle. Sans cette cohérence, les enseignants se trouvent écartelés entre des injonctions contradictoires, ce qui peut déboucher sur des pratiques erratiques et peu efficaces.

D’où devraient provenir les lignes directrices relatives à l'enseignement des mathématiques de base ? Par le passé, des recommandations ont été formulées par des mathématiciens, des didacticiens ou encore des pédagogues sur des bases souvent plus conceptuelles qu'empiriques. Le problème de telles recommandations est qu'elles sont formulées du sommet vers la base, des spécialistes vers les praticiens, ce qui peut entraîner des difficultés d'appropriation par ces derniers. Certains enseignants y adhèrent, mais d'autres s'y opposent parfois radicalement. Par ailleurs, depuis une vingtaine d'années, nos connaissances à propos de la didactique des mathématiques et des processus mentaux à la base des apprentissages mathématiques ont considérablement progressé, qu'il s'agisse de l'appréhension des quantités par les bébés, du rôle de la mémoire dans le calcul mental, de l'impact du langage dans l'apprentissage de la numération de position ou encore des obstacles que peuvent représenter les connaissances antérieures pour de nouveaux apprentissages. Ces connaissances sont encore trop peu prises en compte pour concevoir les curricula, identifier les bases indispensables et concevoir des démarches didactiques mieux adaptées aux capacités des élèves.

Au sein du Conseil national d'évaluation du système scolaire, il est apparu que la définition des lignes directrices pour l'enseignement des nombres et des opérations sur ces nombres ne devait pas être laissée aux mains des seuls spécialistes de la discipline et de son enseignement. Dans la mesure où les connaissances dont il est ici question constituent les compétences mathématiques de base que tout citoyen devrait maîtriser, ces lignes directrices devraient avoir une légitimité beaucoup plus forte. Pour que ce soit le cas, elles devaient être produites par l'ensemble des parties prenantes de l'enseignement des mathématiques de base : les enseignants des différents niveaux scolaires, les parents, les formateurs, les directeurs, les inspecteurs, etc.

Cette manière de procéder caractérise la méthodologie de la conférence de consensus. Cette dernière trouve son origine dans le domaine médical où elle a été souvent utilisée pour définir des " règles de bonne pratique " dans des domaines sensibles où les avis sont divergents et même conflictuels. Elle repose sur un important travail préparatoire qui a pour but d'identifier les questions clés et de dresser un état des lieux des connaissances sur le sujet traité. Ce travail est à la base d'une conférence publique où le bilan des connaissances actuelles est présenté et les questions sont débattues. Un jury d'une vingtaine de personnes participe à l'ensemble de la conférence durant laquelle ses membres ont l'opportunité d'interroger les conférenciers sur toutes les questions sensibles. Les membres du jury sont représentatifs de l'ensemble des parties prenantes des situations problématiques qui ont motivé la conférence de consensus. À l'issue de la conférence, le jury rédige un ensemble de recommandations motivées qui sont les lignes directrices de " bonnes pratiques" dans les situations considérées. Les recommandations sont ensuite diffusées sur le terrain et progressivement mises en œuvre.

Dans le cas présent, huit grandes questions relatives à l'apprentissage des nombres et du calcul ont été identifiées. Des spécialistes des apprentissages numériques, de la didactique et de la pédagogie ont ensuite été invités à dresser un bilan des connaissances actuelles susceptibles d'éclairer ces questions. Une synthèse de leur travail a été présentée lors d'une conférence publique de deux jours à laquelle participait un jury de 18 membres. Ce jury était composé de personnes d'horizons variés 
(enseignants, parents, directeurs, conseiller pédagogique, inspecteurs, formateur, représentant d'association), toutes liées à des degrés divers à la problématique des apprentissages mathématiques.

Les membres du jury ont participé à deux journées préparatoires où ils ont été informés des questions posées et des enjeux de la conférence. À cette occasion, ils ont pu prendre connaissance de plusieurs études scientifiques synthétisant les connaissances actuelles à propos des premiers apprentissages des nombres et des opérations et de la qualité des acquis des élèves dans ces domaines. Lors de ces journées préparatoires, les membres du jury ont également reçu les textes de synthèse des intervenants de la conférence et pu ainsi préparer les questions qu'ils souhaitaient leur poser lors des deux journées de la conférence. Ces deux journées ont permis de présenter un état des lieux très complet des connaissances à propos de l'apprentissage des nombres et des opérations. Cet état des lieux concernait les programmes, les manuels, les pratiques pédagogiques, les performances des élèves, les difficultés d'apprentissage, les liens entre l'apprentissage des concepts et des procédures, les fondements cognitifs des apprentissages, les différences interindividuelles dans l'acquisition des connaissances, la formation des enseignants et les ressources à la disposition de ces derniers. Tout au long des deux journées de la conférence, les membres du jury ont pu interroger les intervenants pour éclairer et approfondir les questions abordées.

Les recommandations présentées dans la suite de ce document ont été rédigées par le jury dans la foulée de la conférence. Les membres du jury ont conscience que leurs recommandations s'appuient sur des connaissances scientifiques variables selon les facettes du domaine abordé. Si, pour certaines facettes, nos connaissances sont aujourd'hui solides et détaillées, pour d'autres facettes, elles restent lacunaires. C'est, par exemple, le cas de nos connaissances à propos de la pertinence et de l'efficacité de certaines ressources pédagogiques et de certaines formations. Le jury encourage dès lors les autorités responsables à stimuler et soutenir les recherches sur plusieurs questions relatives à l'apprentissage des nombres et des opérations. Ces recherches sont une des conditions nécessaires pour améliorer la qualité de l'enseignement et de l'apprentissage des élèves.

Les recommandations du jury constituent des lignes directrices pour un enseignement fructueux des compétences de base en mathématiques. Certaines de ces recommandations sont partiellement mises en œuvre, alors que d'autres supposent une évolution des représentations, des pratiques ou des textes de références. D'autres enfin figurent déjà dans les programmes du Conseil supérieur des programmes, et le jury a souhaité les intégrer dans ce document pour attirer l'attention des praticiens qui liront les recommandations. 


\section{Composition du jury de la conférence de consensus}

Le jury est présidé par Jacques GRÉGOIRE, docteur en psychologie, professeur à l'université catholique de Louvain. Il est composé des personnalités suivantes :

Marion ALVINIERIE

Julien BELGHITI

Patricia BORGNA

Nawel BOSSY-SEMMOUD

Nathalie CHALARD

Marie-Claude CORTIAL

Marc DIETRICH

Emmanuel FRITSCH

Sophie JALLOT-LABAS

Jean LABBOUZ

Stéphane MARCHAND-ADAM

Laurent NOÉ

Stéphane PIERRE

Frédérick TEMPIER

Claire TORRES BISQUERRA

Christophe TOURNEUX

Anne VALENTIN
Professeure des écoles stagiaire

Professeur de Lycée Professionnel Mathématiques et Sciences

Professeure des écoles

Conseillère pédagogique du $1^{\mathrm{er}}$ degré en Mathématiques et Sciences

Professeure de mathématiques

Présidente du groupe Éducation et Devenir (jusqu'en octobre 2015)

Professeur des écoles

Parent d'élèves

Professeure des écoles

Inspecteur de l'Éducation nationale Mathématiques et Sciences

Directeur d'école maternelle

Directeur académique des services de l'Education nationale (DASEN) adjoint de Seine St Denis

Inspecteur de l'Education nationale, chargé de circonscription du premier degré

Maître de conférences à l'université Cergy-Pontoise, formateur en ÉSPÉ

Parent d'élèves

Inspecteur d'académie - inspecteur pédagogique régional (IA-IPR) de mathématiques

Enseignante spécialisée dans le $1^{\text {er }}$ degré 


\section{Principes généraux pour un meilleur enseignement des nombres et du calcul à l'école primaire}

\section{Que nous dit la recherche sur les apprentissages?}

L'apprentissage des nombres et du calcul est une activité complexe qui repose sur de nombreuses compétences (cognitives, langagières, visuo-spatiales). Les travaux de recherche présentés lors de la conférence, notamment dans le champ de la psychologie développementale et cognitive, ont identifié trois grands moments clés de cet apprentissage, qui surviennent plusieurs fois dans la scolarité d'un élève: avec les nombres entiers au début de l'école, puis avec les nombres fractionnaires et décimaux. Ces trois moments clés sont :

- la conception des nombres, c'est-à-dire le passage d'un traitement intuitif et approximatif des grandeurs et des quantités à un traitement exact des nombres ; ce passage concerne essentiellement l'acquisition des premiers nombres chez les jeunes enfants, mais d'autres découvertes, comme l'existence de nouveaux nombres entre deux nombres entiers, interviennent plus tard ;

- la désignation des nombres dans un langage spécifique (oral) et dans un système universel (écrit) ; pour les nombres entiers, cette étape doit satisfaire les irrégularités de la numération orale "à la française " (onze, soixante-dix, quatre-vingts), puis les codes de la numération écrite (groupements par 10, importance de la position des chiffres, rôle du "zéro ") qui permettent, grâce à l'introduction d'un nouveau symbole, la virgule, d'écrire les nombres décimaux ;

- I'utilisation d'opérations arithmétiques sur les nombres pour résoudre des problèmes ; l'emploi de symboles permet d'aboutir aux mêmes résultats que la manipulation concrète d'objets, il est même plus rapide grâce à certaines propriétés, et il rend possible le traitement de situations difficiles ou impossibles à matérialiser.

Si les trois points évoqués ci-dessus apparaissent comme des objectifs des programmes scolaires en France, chacun d'entre eux présente des difficultés d'apprentissage mises en évidence par la recherche, et tous les élèves ne les acquièrent pas à l'issue de l'école primaire. La recherche a aussi montré que des différences de cheminements cognitifs existent chez les enfants, que tous les enfants ne font pas appel aux mêmes composantes pour apprendre, et elle a permis de comprendre les troubles qui gênent l'apprentissage de certains d'entre eux. Comment alors un enseignant peut-il intégrer dans l'exercice de son métier au quotidien ces différences ? 


\section{Conséquences transversales pour l'enseignement des nombres et du calcul}

À l'heure actuelle, la recherche dans le domaine de l'enseignement des nombres et du calcul propose des éléments de compréhension des premiers apprentissages numériques, mais reste très fragmentaire à propos de l'efficacité des méthodes d'enseignement des nombres et des opérations. En effet, les études empiriques, menées dans des classes, et qui permettraient d'identifier des pratiques "efficaces" restent peu nombreuses. Toutefois, les résultats d'études diverses et la confrontation d'expériences de terrain permettent de dégager quelques grands principes susceptibles d'orienter le travail des enseignants en amont des recommandations :

1. les élèves ont des connaissances qui proviennent de capacités innées et des expériences de la vie quotidienne (sens des quantités, ou des grandeurs comme la monnaie ou les longueurs, ou encore la notion de partage, d'un gâteau par exemple) ;

2. le langage oral est essentiel dans la désignation et le passage à la symbolisation des nombres ${ }^{3}$ (entiers, décimaux,...) ;

3. un formalisme prématuré nuit à la compréhension des nombres; le recours à la manipulation et à l'expérimentation ne concerne pas seulement l'école maternelle, mais doit s'étendre à l'école élémentaire ;

4. I'acquisition de faits numériques ${ }^{4}$ et d'automatismes est nécessaire ;

5. l'acquisition de procédures (ex : effectuer une soustraction) et leur compréhension sont complémentaires ;

6. les activités cognitives impliquées dans le calcul mental et par le calcul effectué par écrit (calcul posé) ne sont pas de même nature ${ }^{5}$;

7. le report de l'enseignement de notions qui apparaissent difficiles aux enseignants (par exemple celui des décimaux qui est souvent reporté à la fin du CM1) et un temps d'enseignement réduit de ces mêmes notions dessert les élèves ;

8. I'utilisation de méthodes et de matériaux diversifiés est un moyen de répondre à la variété des cheminements d'apprentissage et de développement; les textes de savoir (ce que les élèves ont à retenir) sont des moyens de rassembler et de synthétiser les processus individuels d'apprentissage et l'histoire collective de la classe.

\footnotetext{
3 Les élèves doivent assimiler différentes conventions de langage qui dépendent des cultures : on dit vingt-cinq en Français mais fünfundzwanzig (littéralement cinq et vingt) en Allemand. En outre, ces règles opposent des combinaisons de types additif et multiplicatif : par exemple, 108, c'est "cent et huit ", alors que 800, c'est " huit fois cent ».

${ }^{4}$ La connaissance des tables d'addition et de multiplication, mais pas seulement; par exemple, des relations multiplicatives simples entre les nombres (30 est le double de 15) ou l'association de deux écritures différentes d'un même nombre $(1 / 2=0,5)$.

5 Le calcul posé repose sur des procédures standards, il fonctionne sur les chiffres, et il exige de travailler « de droite à gauche ", alors que le calcul mental a un fondement heuristique, il opère sur les nombres au lieu des chiffres, et va « de gauche à droite ».
} 


\section{Recommandations générales}

R1 - Les mathématiques doivent être présentées aux élèves comme des outils pour penser, résoudre des problèmes et faire face à des situations de la vie quotidienne.

Commentaires : Trop souvent les mathématiques apparaissent d'abord comme des savoirs élitistes utilisés pour la sélection et la promotion. Elles ne sont perçues ni comme des activités intellectuelles ludiques ni comme des connaissances et des savoir-faire que devraient posséder tous les citoyens. L'utilité et le sens des notions et des procédures enseignées à l'école primaire devraient être manifestes tout au long de la formation mathématique des élèves.

R2 - La continuité et la cohérence de l'enseignement des mathématiques au travers des années, des cycles et des degrés doivent permettre aux élèves de construire des savoirs et savoir-faire qui s'enchaînent et s'intègrent harmonieusement tout au long de leur cursus d'apprentissage.

Commentaires : Les programmes doivent mettre de façon centrale l'accent sur la logique du cursus mathématique, la nécessaire intégration des connaissances tout au long du parcours scolaire de l'élève et l'approfondissement progressif des concepts dans un processus d'apprentissage en spirale $^{6}$. Les recherches montrent que de nombreux enseignants sont isolés dans leur classe, sans avoir une connaissance claire de ce qui a été enseigné dans la classe précédente et de ce qui le sera dans la classe suivante. Le manque de continuité dans les apprentissages peut être source d'incohérence et d'inefficacité de l'enseignement des mathématiques. Le travail en équipe d'enseignants de différents niveaux et de différents cycles doit effectivement pouvoir être mis en œuvre et valorisé par l'institution pour permettre une cohérence et une continuité pédagogique favorable aux apprentissages des élèves.

\footnotetext{
${ }^{6}$ Une progression en spirale se caractérise par une organisation de l'enseignement qui permet aux élèves de fréquenter une même notion plusieurs fois dans l'année scolaire, avec des degrés d'approfondissement et de formulation adaptés à leurs niveaux d'apprentissage. Elle se distingue d'une formation par chapitres ou par micro-chapitres.
} 


\section{Recommandations relatives à l'enseignement à l'école maternelle}

R3 - Les premiers apprentissages en mathématiques doivent pouvoir prendre appui sur des capacités que les enfants possèdent avant leur scolarisation.

Commentaires: Les recherches montrent que les enfants en bas âge possèdent déjà certaines capacités numériques ${ }^{7}$ qui peuvent être valorisées par les enseignants pour construire des capacités plus larges et plus élaborées.

R4 - La compréhension du concept de nombre s'appuie sur les compétences cognitives (verbales, visuo-spatiales, mnésiques...) qui doivent être développées en classe.

Commentaires: La compréhension en mathématiques nécessite l'apprentissage d'un vocabulaire spécifique (plus que / moins que/autant que...) et une connaissance de la chaîne numérique verbale. La maîtrise de ces connaissances verbales facilite la compréhension des situations mathématiques. Différentes études ont mis en évidence un lien entre le développement des capacités verbales et les compétences numériques, ainsi que l'importance de la dimension spatiale dans l'acquisition de ces dernières.

R5 - Les premiers apprentissages mathématiques doivent reposer sur des manipulations d'objets variées et répétées dans une visée progressive de symbolisation et d’abstraction.

Commentaires : Les manipulations sans conceptualisation ne permettent pas d'atteindre les objectifs des apprentissages numériques. Les manipulations doivent soutenir la construction progressive de représentations mentales des nombres au départ de la désignation, de la reproduction et de la mise en correspondance de collections d'objets variées. La diversité des situations proposées permet une variété dans les chemins empruntés par les élèves pour construire la représentation des nombres et des opérations. Les situations de manipulation permettent de développer des habiletés spatiales, mais aussi langagières spécifiques. Les jeux faisant appel aux nombres et aux opérations sont la forme privilégiée de ces manipulations.

\footnotetext{
${ }^{7}$ Ces capacités de base ${ }^{7}$ permettraient par exemple une estimation approximative et une comparaison rapide des grandeurs et quantités ainsi que la perception des ajouts ou retraits et de leurs effets.
} 
R6 - Les enseignants doivent comprendre la complexité des processus mis en œuvre dans le dénombrement de collections par l'enfant et utiliser ces situations comme des moments privilégiés d'identification de difficultés d'apprentissage.

Commentaires : Le dénombrement d'une collection implique des compétences verbales, visuelles, motrices et conceptuelles. De multiples difficultés peuvent apparaître dans l'exercice coordonné de ces compétences, ce qui aboutit à des dénombrements erronés et inconstants et, par conséquent, un problème dans le développement de représentations numériques précises. II est dès lors important de repérer les difficultés de dénombrement et d'aider l'enfant à mettre en place une procédure correcte. II importe que le dénombrement soit une procédure réflexive. L'élève doit comprendre pourquoi certaines règles de dénombrement (par exemple, pointer une seule fois chaque objet) sont essentielles, alors que d'autres démarches n'influencent pas le résultat du dénombrement (par exemple, dénombrer de gauche à droite ou de droite à gauche aboutit au même résultat).

R7 - Lors de l'apprentissage des mots désignant les nombres, il importe de les associer à différentes représentations.

Commentaires: II est nécessaire de systématiser le recours à des représentations numériques variées associées à la suite numérique verbale. La différence entre chiffres (symboles indo-arabes) et nombres doit être explicitée. Les représentations analogiques devraient être privilégiées (utilisation de collections d'objets variés), les symboles écrits $(1,2,3 \ldots)$ étant proposés aux enfants à partir de 4 ans. Les formulations orales telles que « un...et un...deux, et un....trois » font partie de ces activités.

R8 - Les pratiques régulières et variées de composition/décomposition de petites collections doivent être favorisées, car elles permettent de donner du sens aux nombres et d'approcher les notions d'addition et de soustraction.

Commentaires : Les activités de composition/décomposition (par exemple, découvrir que 5 c'est 2 et 3 , mais aussi 1 et 4 , etc.) permettent de donner du sens aux nombres comme des classes sériées (3 est plus petit que 4, qui est plus petit que 5...) et emboîtées ( 3 est inclus dans 4 qui est inclus dans 5...). Elles développent aussi l'acquisition d'une aisance dans la manipulation et les procédures. Elles favorisent la mémorisation des premiers faits numériques (premiers éléments des tables d'additions et de soustractions et en particulier la liste des compléments à 10) et l'acquisition de techniques de calcul. 


\section{Recommandations relatives à l'enseignement à l'école élémentaire}

R9 - L'enseignement des nombres et des opérations nécessite de faire progressivement comprendre ce que sont les nombres et les opérations et à quelles questions ils permettent de répondre.

Commentaires : Les observations réalisées dans les classes montrent qu'à la fin de l'école primaire, les élèves réussissent des tâches, sans maîtriser les connaissances conceptuelles associées. L'enseignement des nombres et des opérations ne peut se limiter à l'enseignement de leur utilisation. Par exemple, apprendre à effectuer une opération ne permet pas de comprendre à quoi sert cette opération, écrire un nombre décimal sous différentes formes peut se révéler n'être qu'un exercice purement technique qui ne permet pas de mieux comprendre ce qu'est un nombre décimal. Enseigner les nombres, entiers ou décimaux ,nécessite un travail important, organisé progressivement dans la durée, sur la compréhension du sens de ces notions, sur les questions auxquelles ces types de nombres permettent de répondre (étudier par exemple des situations qui motivent le recours à des nombres autres que les entiers), sur les différents aspects inhérents à ces notions (par exemple : cardinal, pour exprimer des quantités, et ordinal, pour exprimer le rang dans une liste, pour les entiers, fraction représentant le fractionnement de l'unité ou, plus tard, fraction à concevoir comme un quotient,...), sur la compréhension des différentes écritures des nombres, en particulier pour les nombres décimaux et les fractions, ...

R10 - L'enseignant doit être attentif au fait que des compétences langagières et visuo-spatiales déficientes peuvent entraver l'acquisition des compétences numériques et des opérations sur les nombres. Varier les situations mathématiques et les modes de représentation du nombre permet de prendre en compte la variété des compétences et des styles cognitifs des élèves.

Commentaires : Différentes recherches montrent que les compétences langagières et spatiales interviennent dans la construction du nombre, notamment celles menées auprès d'élèves présentant des troubles "dys " (dyscalculie, dyspraxie, dysphasie ...). Notre façon de dire les nombres en français s'appuie sur un système structuré, mais présentant de nombreuses irrégularités, en particulier pour les nombres inférieurs à cent. L'écriture des nombres en chiffres met en jeu des connaissances spatiales liées à la nature positionnelle de ce système. 
R11 - L'acquisition du système de numération décimale de position est fondamentale pour les apprentissages numériques.

Commentaires : Cet enseignement ne se limite pas à apprendre à écrire et à dire les nombres, mais $s^{\prime}$ attache à permettre une compréhension des aspects décimal et positionnel ${ }^{8}$. La maîtrise de ce système de numération passe par la manipulation exercée des nombres supérieurs à 100, faute de quoi l'élève ne peut accéder à la signification de la position des chiffres dans le nombre. Des recherches empiriques ont montré que la réussite dans l'apprentissage des décimaux est conditionnée par une bonne connaissance des nombres entiers. Les évaluations nationales renforcent ce constat : elles font apparaître des difficultés sur les décimaux dont on peut penser qu'elles sont le signe d'une construction insuffisante des nombres entiers (par exemple, les réponses erronées « $0,24>0,5$ « ou « $0,2<0,10$ » indiquent une compréhension incomplète du système décimal et de position).

R12 - L'étude des fractions précède celle des nombres décimaux, mais doit se limiter aux fractions simples (demi, tiers, quart...) et aux fractions décimales (dixièmes, centièmes...) dans le cas du fractionnement de l'unité.

Commentaires : Des travaux de recherche en didactique et en psychologie des apprentissages montrent l'utilité de s'appuyer sur les fractions pour donner du sens aux nombres décimaux, mais aussi que le traitement et la compréhension des fractions sont particulièrement difficiles pour les élèves. Dès lors, cet apprentissage ne doit pas être trop ambitieux à l'école primaire. Il sera limité à une maîtrise du fractionnement de l'unité en parts égales sur les fractions simples puis sur les fractions décimales (dixième, centième, ...) permettant la compréhension de la signification des chiffres dans l'écriture à virgule.

R13 - Le système d'écriture des nombres décimaux est un prolongement de celui des nombres entiers. L'identification de cette continuité doit être présentée de manière explicite auprès des élèves, tout en attirant l'attention des élèves sur certaines adaptations nécessaires.

Commentaires : Les principales erreurs des élèves dans l'apprentissage des nombres décimaux sont dues à l'utilisation de règles valables pour les nombres entiers qui deviennent erronées quand elles sont transférées telles quelles aux nombres décimaux.

\footnotetext{
8 Le système de numération est dit positionnel car la valeur des chiffres dépend de leur position (par exemple, dans « 14 », le chiffre " 1 » représente une dizaine, alors que le chiffre " 4 » représente quatre unités) .Ce système est appelé décimal car la représentation symbolique des nombres est basée sur les puissances de 10 (par exemple, « 1 » dans le rang des dizaines représente 10 unités et « 1 » dans le rang des centaines représente 10 dizaines).
} 
R14 - Bien qu'il existe des outils informatiques de calcul très performants, le calcul mental et le calcul posé doivent continuer à occuper une place importante dans l'enseignement des mathématiques.

Commentaires : Outre le fait que cet apprentissage permet de développer les stratégies efficientes de calcul (par exemple, calculer à partir du plus grand, décomposer en dizaines et unités, transformer la soustraction en addition...), il permet d'approfondir les notions de nombre et d'opération et permet une entrée progressive dans l'abstraction.

R15 - L'enseignement du calcul avec les nombres entiers et décimaux devrait associer l'apprentissage des techniques opératoires à celui du sens des opérations. II est important de développer l'intelligence du calcul en lien avec une compréhension profonde de la notion de nombre.

Commentaires : Travailler le sens des opérations, c'est, par exemple, dans le cas de la résolution de problèmes, rendre l'élève capable de choisir une opération adaptée au traitement d'une situation. Ainsi, un élève devrait avoir la possibilité, en fonction de ses préférences, de traiter une soustraction en s'appuyant d'abord sur l'addition. II devrait également, par un apprentissage progressif, être en mesure de choisir la stratégie et le mode de calcul (calcul mental, en ligne, posé ou instrumenté) les plus adaptés à la situation. Les procédures de calcul posé, pour la soustraction en particulier, peuvent ou non favoriser la compréhension de la numération de position; il convient de privilégier l'enseignement de celles qui amèneront l'élève à mieux comprendre plutôt que celles qui ne visent que l'efficacité du traitement.

R16 - L'enseignement du calcul, avec les nombres entiers et décimaux, doit permettre la découverte, la compréhension progressive, l'appropriation, puis la mobilisation des propriétés des opérations.

Commentaires : Par exemple, travailler le fait que : $6,7 \times 5=5 \times 6,7$ donne plus facilement accès, dans le traitement de $6,7 \times 5$ à des procédures utilisant la décomposition de 6,7 en $6+0,7$. En l'occurrence, $6,7 \times 5=5 \times 6,7=5 \times(6+0,7)=(5 \times 6)+(5 \times 0,7)$.

Apprendre par exemple que $(6 \times 4) \times 25=6 \times(4 \times 25)$ donne accès à des procédures efficaces pour effectuer $24 \times 25$. Amener les élèves à explorer les diverses décompositions $d$ 'un nombre, sous la forme d'une somme ou d'un produit par exemple, est dans ce cadre un élément facilitant. Remarquer que $153-78=155-80=175-100$ et amener les élèves à dire qu'ajouter un même nombre aux deux nombres qui composent une soustraction ne change pas son résultat, permettra de les rendre capables d'effectuer de façon beaucoup plus aisée, mentalement ou dans un calcul écrit en ligne : 153-78. Cet entraînement à la flexibilité mentale et à la verbalisation des stratégies pourrait être utile à tous et en particulier aux élèves ayant des difficultés d'apprentissage. 


\section{R17 - Le calcul mental et le calcul en ligne doivent être privilégiés par rapport au calcul posé.}

Commentaires : Le calcul mental et le calcul en ligne devraient être travaillés avant le calcul posé. L'enseignant devrait leur consacrer plus de temps qu'au calcul posé sur des périodes plus longues. L'enseignement du calcul mental et celui du calcul posé prévoient de travailler conjointement l'enrichissement des répertoires (mémorisation de tables et faits numériques, "sous-procédures "), et le traitement de calculs complexes nécessitant la mobilisation successive de diverses "sousprocédures ". II ne s'agit pas de supprimer le calcul posé de nombres à plusieurs chiffres; ce type de calcul peut en effet représenter une tâche complexe mettant en jeu plusieurs calculs mentaux successifs.

R18 - L'enseignement du calcul mental et du calcul en ligne doit être organisé selon une progressivité.

Commentaires : Cet apprentissage repose sur : (1) la prise en compte des besoins identifiés des élèves, de leurs différents cheminements et de leurs erreurs, (2) une pratique fréquente et la gradation de la difficulté, (3) la maîtrise des faits numériques (les tables des opérations, les compléments à 10 et à 20 , et quelques multiples remarquables...), (4) le développement de capacités d'adaptation en fonction de la nature et de la valeur des nombres, (5) le développement de procédures de contrôle (évaluation des ordres de grandeurs), et (6) la nécessité d'une trace écrite explicitant les différents savoirs rencontrés.

R19 - L'enseignement du calcul mental et du calcul en ligne doit donner une place importante à la verbalisation par les élèves de leurs façons de faire, qu'elles soient correctes ou non.

Commentaires: II est essentiel que l'élève identifie clairement les procédures fiables. Une trace écrite, exprimée en des termes accessibles à tous les élèves, permet de garder en mémoire les savoirs rencontrés et les procédures abouties et correctes mises en œuvre.

R20 - Les élèves doivent apprendre à utiliser le calcul mental ou le calcul en ligne pour déterminer l'ordre de grandeur d'un résultat afin de le contrôler ou, de façon plus générale, pour effectuer un calcul approché.

Commentaires : Le calcul mental et le calcul en ligne peuvent ainsi constituer un moyen de contrôle du calcul exact, qu'il soit effectué en calcul posé ou à la calculatrice. Par ailleurs, le contexte de diverses situations problèmes n'impose pas l'obtention d'un résultat exact, un résultat approché obtenu mentalement peut parfois être suffisant; progressivement, il convient que les élèves puissent, de façon autonome, traiter ainsi de telles situations. 
R21 - Les opérations sont introduites par la résolution de problèmes.

- R21.1 - Les situations relevant de l'addition et de la soustraction sont travaillées de manière quasi simultanée; il en est de même des situations relevant de la multiplication et de la division.

- R21.2 - Les problèmes proposés appartiennent aux différentes catégories de situations d'addition/soustraction et de multiplication/division afin de permettre à l'élève de reconnaître les différents modèles.

Commentaires : La recherche montre par exemple que les problèmes soustractifs proposés aux élèves correspondent trop souvent à des situations de retrait. II convient en conséquence de travailler aussi les situations d'écart. Il est nécessaire de varier les problèmes rencontrés et d'en expliciter les points communs afin de construire des catégories générales de problèmes. 


\section{Recommandations relatives à la formation initiale et continue des enseignants}

R22 - Les recommandations citées dans les autres sections doivent être prises en compte dans le cadre de la formation initiale et continue des enseignants en mathématiques et en didactique des mathématiques.

Commentaires: Au-delà de la nécessaire maîtrise des contenus théoriques, et notamment en mathématiques, les formateurs et didacticiens seront attentifs aux conceptions initiales des futurs enseignants vis-à-vis des mathématiques. La numération de position et notre système décimal seront étudiés de façon à leur faire prendre conscience des obstacles et de la nécessaire prise en compte des différents cheminements que peuvent avoir les élèves. Les enseignants doivent avoir les éléments de compréhension des fondements et de la logique des nouveaux programmes et des nouveaux documents d'accompagnement.

R23 - La formation initiale et la formation continue doivent prendre en compte l'ensemble des savoirs et des savoir-faire nécessaires pour pouvoir enseigner efficacement des connaissances de base en mathématiques.

Commentaires: Les difficultés d'apprentissage des élèves peuvent parfois être provoquées par certains procédés d'enseignement inadéquats. La formation devrait permettre d'identifier ces phénomènes et de donner des moyens pour les dépasser. Les compétences essentielles pour enseigner les bases des mathématiques concernent: (1) les concepts mathématiques et leurs fondements, (2) les principes de la didactique des mathématiques, (3) les fondements cognitifs et émotionnels des apprentissages mathématiques, et (4) les différences interindividuelles dans l'apprentissage des mathématiques.

R24 - La conception des formations doit intégrer les différentes facettes des savoirs et savoir-faire à maîtriser par les enseignants. Pour cela, des équipes pluridisciplinaires de formateurs (chercheurs, formateurs et enseignants) doivent être mises en place.

Commentaires: Une formation efficace, c'est-à-dire qui permette de faire évoluer les représentations et les pratiques, doit s'appuyer sur des compétences rarement rassemblées chez une même personne. Ces compétences concernent les connaissances issues des recherches en psychologie et en didactique, l'expérience du terrain (les obstacles et les ressources), et la conception et l'organisation de formations. Il apparaît dès lors utile de faire travailler ensemble les différentes personnes possédant une ou plusieurs de ces compétences. 
R25 - Les actions de formation tout au long de la carrière doivent être encouragées, notamment lors de l'arrivée de nouveaux textes institutionnels (programmes de mathématiques et documents d'accompagnements) et en fonction de l'avancée des connaissances en psychologie et en didactique des mathématiques.

Commentaires: Une différence doit être faite entre les nouveaux enseignants, qui ont besoin d'être confortés sur les programmes, les savoirs-clés (.....) et leur progression par cycle, et les enseignants expérimentés à la recherche de thèmes nouveaux, de nouvelles organisations des savoirs qui leur permettent d'adapter leurs pratiques pédagogiques. 


\section{Recommandations relatives aux ressources pour la classe mises à la disposition des enseignants}

Le développement du numérique a multiplié et diversifié les ressources mises à la disposition des enseignants. Ces derniers peuvent aujourd'hui se référer à bien d'autres sources d'information que les traditionnels manuels scolaires. Mais il ne suffit pas que des ressources soient à la disposition des enseignants, encore faut-il que celles-ci soient correctement exploitées et accompagnées dans leur mise en œuvre afin de réellement favoriser les apprentissages mathématiques. Mal conçues et/ou mal utilisées les ressources pédagogiques peuvent être nuisibles en stimulant le développement de représentations et de procédures erronées qui peuvent être des obstacles pour les apprentissages futurs.

R26 - Le ministère chargé de l'Éducation doit mettre à la disposition des enseignants des ressources riches et finalisées pour un usage possible en classe, alternatif ou complémentaire à l'utilisation de manuels et fichiers.

Commentaires : Ces ressources doivent pouvoir être adaptées par chaque enseignant au contexte de sa classe et aux spécificités de ses élèves. Elles doivent présenter les caractéristiques suivantes :

- éviter la segmentation et apporter de la cohérence dans l'apprentissage des notions,

- valoriser la progressivité des apprentissages et notamment de l'organisation en cycles,

- tenir compte de la diversité des profils des élèves. Dans cette optique, des situations et contextes d'apprentissages différents devraient être proposés afin de tenir compte des styles d'apprentissages et de la variété des chemins d'apprentissage mis en évidence par la recherche,

- contenir des outils et des recommandations pour l'évaluation diagnostique, formative et sommative des élèves,

- proposer des textes de savoirs (synthèse de ce qu'il y a lieu de retenir d'une séquence d’apprentissage) adaptés à la compréhension des élèves.

R27 - Les sites institutionnels doivent renseigner et documenter une diversité de ressources utiles aux apprentissages sur les nombres et les opérations, et fournir les informations nécessaires pour leur usage pédagogique adéquat : jeux de plateau, jeux en ligne, didacticiels, banques d'images ou de vidéos, ...

Commentaires : Parmi ces ressources, celles pouvant être utilisées dans le cadre familial doivent être signalées. Le recours aux ressources en ligne par les familles est une réalité. Cette pratique doit pouvoir s'inscrire dans une continuité avec le travail conduit à l'école. 
R28 - La mutualisation et le travail d'équipe doivent être renforcés et reconnus, tant pour la conception de ressources que pour leur utilisation cohérente au sein d'une école ou d'un réseau écoles-collège.

Commentaires : Chaque cycle doit contribuer à l'acquisition du socle commun de connaissances, de compétences et de culture par tous les élèves. Dans cette perspective, il est nécessaire de mettre les enseignements des différentes années et des différents cycles en harmonie et d'encourager la cohérence des enseignements non seulement au sein de l'école, mais également entre l'école et le collège. Les concertations entre enseignants devraient permettre d'assurer la continuité des apprentissages aux différents niveaux d'enseignement et d'éviter de mettre en difficulté les élèves les plus fragiles par des ruptures dans le continuum des enseignements.

R29 - Le transfert des résultats de la recherche dans le domaine de l'enseignement des nombres et des opérations doit être favorisé.

Commentaires : L'émergence de collectifs de travail en contact avec des formateurs et chercheurs doit être encouragée dans le cadre de l'accompagnement de projets. La présence dans les écoles d'étudiants et de professeurs stagiaires engagés dans des démarches de recherche constitue une opportunité.

R30 - Du matériel et des référentiels mathématiques adaptés à la maternelle doivent être mis à la disposition des enseignants.

Commentaires : Trop peu d'outils et de documents pédagogiques sont élaborés à destination des enseignants de maternelle, tenant compte des spécificités des jeunes enfants et des apprentissages numériques à leur niveau.

R31 - Les enseignants doivent être attentifs à fournir aux parents des informations concrètes et argumentées pour les aider à soutenir leurs enfants dans les apprentissages des nombres et des opérations.

Commentaires: De grandes inégalités existent du point de vue du soutien familial aux apprentissages scolaires. De nombreux parents, qui ont parfois éprouvé eux-mêmes des difficultés scolaires, sont démunis particulièrement dans le domaine des mathématiques. D'un autre côté, il n'est pas réaliste d'attendre de l'école qu'elle compense tout ce qui ne se fait pas, ou trop peu, à la maison.

Des suggestions de jeux et d'activités données aux parents dans le cadre d'un échange constructif permettraient de stimuler, développer et renforcer un certain nombre de connaissances et de procédures utiles pour les apprentissages numériques : jouer en famille à des jeux de plateau, faire participer l'enfant à des tâches "ménagères" nécessitant l'anticipation, le comptage, la catégorisation comme, par exemple, mettre la table, trier les couverts et les jouets, utiliser la monnaie, se familiariser avec le calendrier et la pendule, réaliser des pesées d'ingrédients lors d'activités de cuisine... 


\section{Recommandations relatives aux programmes}

R32 - Les programmes relatifs aux nombres et au calcul doivent contenir des éléments explicitant les intentions et justifiant les choix qui les fondent.

Commentaires: Il est important de justifier les fondements rationnels et empiriques des programmes pour qu'ils aient du sens et une légitimité pour les différents acteurs. Les arguments peuvent être d'ordre didactique, peuvent concerner des applications de la vie quotidienne, la suite logique des apprentissages, l'évolution des outils informatiques, les résultats de la recherche, etc.

\section{R33 - Une évaluation systématique des programmes doit être mise en place.}

Commentaires : L'évaluation permet de faire évoluer les programmes en prenant en compte (1) les acquis des élèves mesurés lors des enquêtes nationales et internationales, (2) la difficulté et les errements de leur mise en œuvre par les enseignants et (3) les résultats des recherches en didactique des mathématiques et en cognition numérique. L'évaluation des programmes doit non seulement interroger le bien-fondé de l'enseignement de certaines notions et procédures, mais aussi l'enchaînement et le rythme des apprentissages, ainsi que leur adéquation au niveau de développement des élèves. 


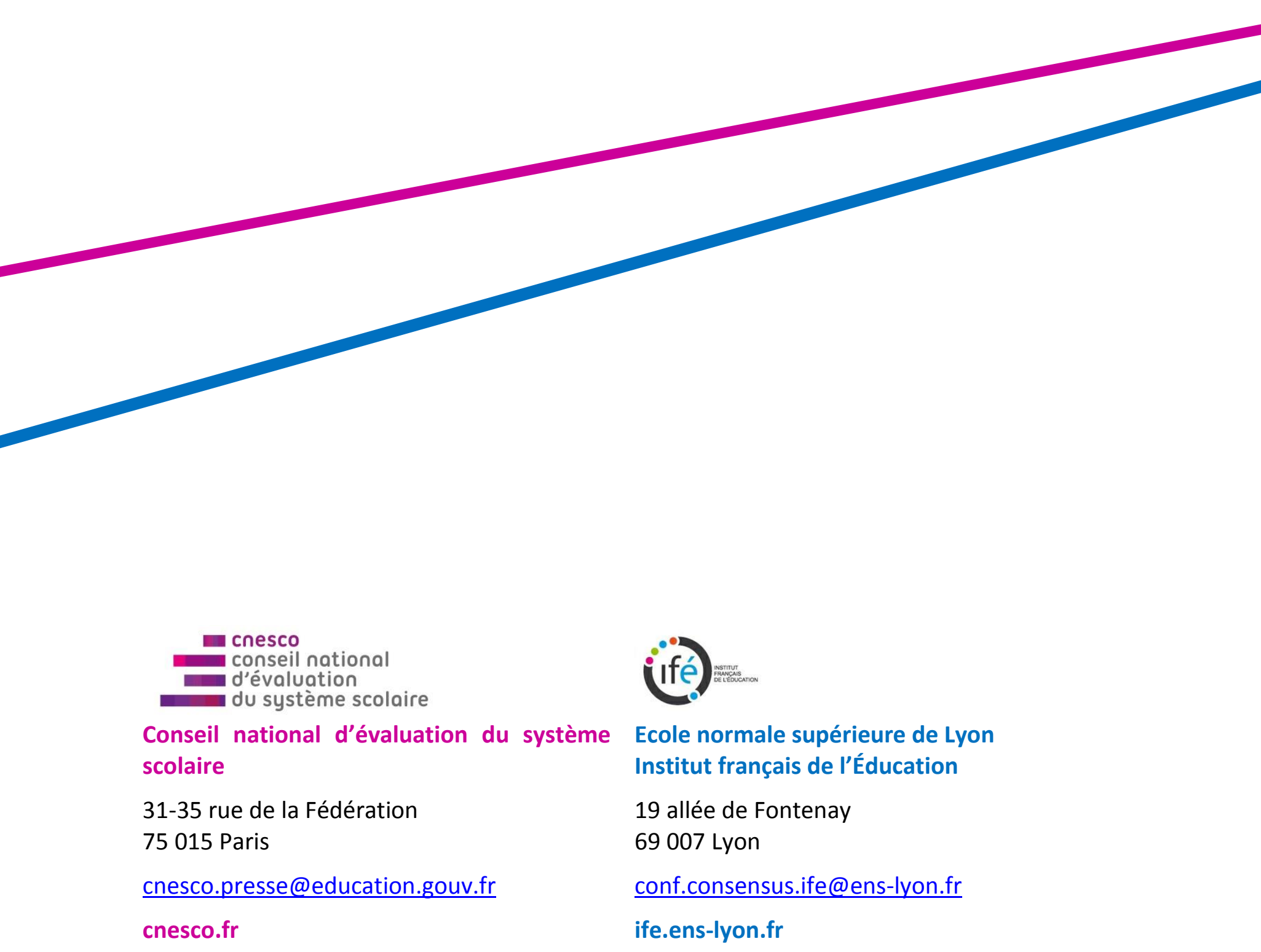

